\title{
A Critique of Popper's Views on Scientific Method
}

\author{
Nicholas Maxwell University College London \\ (First Published in Philosophy of Science, vol. 39, no. 2, June 1972, pp. 131-152; reprinted in \\ Popper: Critical Assessments of Leading Philosophers, Vol. II, Part 3, edited by Anthony \\ O'Hear, Routledge, London, pp. 463-487)
}

This paper considers objections to Popper's views on scientific method. It is argued that criticism of Popper's views, developed by Kuhn, Feyerabend, and Lakatos, are not too damaging, although they do require that Popper's views be modified somewhat. It is argued that a much more serious criticism is that Popper has failed to provide us with any reason for holding that the methodological rules he advocates give us a better hope of realizing the aims of science than any other set of rules. Consequently, Popper cannot adequately explain why we should value scientific theories more than other sorts of theories; which in turn means that Popper fails to solve adequately his fundamental problem, namely the problem of demarcation. It is suggested that in order to get around this difficulty we need to take the search for explanations as a fundamental aim of science.

\section{I}

In this paper my aim is to discuss some of the more serious difficulties that Popper's theory of scientific method runs into.

In recent years a number of criticisms of Popper's views have been developed by Kuhn, Feyerabend, Lakatos, and others. I shall argue that these criticisms, in so far as they are valid, follow a common pattern, and can be seen as special cases of a rather more general criticism of Popper. I shall argue that this general type of criticism of Popper is not in fact too damaging, although it does require that Popper's views be modified somewhat.

There is, however, a rather different criticism to be made of Popper's theory, which is much more serious. It amounts to the claim that Popper has failed to provide a rationale for the methodological rules he advocates, and has thus failed to provide an adequate solution to his fundamental problem - namely the problem of demarcation.

Before going any further, I should like to say that, despite my criticisms, I have the greatest admiration for Popper's writings on scientific method ([8], [9], and [10]). I am in complete sympathy with his basic intentions and values. My hope in criticizing Popper's theory is to clarify the problems which confront the theory so that we may have a clearer idea of how the theory can be strengthened and improved so as to cope with these problems.

II

The heart of Popperian methodology may be expressed like this. As scientists, in our hopeful search for the Truth, in our attempt to solve problems of ever increasing profundity, we put forward wild, improbable conjectures, of ever increasing empirical content and explanatory power, which we then seek to overthrow by subjecting them to as severe experimental testing as possible. At any given stage the best theory is the theory of highest empirical content which has stood up best to all our attempts at experimental refutation.

The methodological rules advocated by Popper-governing the acceptance and rejection of 
theories in science-are designed to give us the best hope of realizing the above aims. According to Popper a supreme rule may be laid down governing the choice of other methodological rules. It is the rule "which says that the other rules of scientific procedure must be designed in such a way that they do not protect any statement in science against falsification" ([8], p. 54).

One point — about which Popper himself has been a bit equivocal—must be made straight away. Scientific theories cannot be refuted experimentally with absolute certainty. This is due in part to Duhem's point that it must always be a group of theories that is tested experimentally, individual theories strictly being incapable of being tested (see [2], Ch. VI). But more generally, it is due to the fact that in order to refute with certainty any scientific theory we must establish with certainty the truth of some falsifying hypothesis-and, this, we may take it, cannot be done. It is thus always a conjecture that a theory, which our methodological rules leads us to reject, has in fact been found to be false. There is, in other words, always the possibility that the application of Popperian rules may lead us to reject a theory which is in fact true. This situation is not, however, too disastrous - as long as it is reasonable to hold that Popperian rules give us a good hope of correctly detecting error in our theories.

Popper has been at pains to emphasize that a theory can only be falsified with respect to the application of methodological rules (see for example [8], pp. 81-2). But this formulation of the issue obscures the decisive point - that, for all we know, application of Popperian rules may lead us again and again to reject true theories. Popper speaks as if we know somehow that Popperian rules cannot lead us to reject true theories.

I turn now to a consideration of some of the criticisms that have been made of Popper's theory.

In the first place it has sometimes been argued, in effect, that Popperian rules are not, or have not been, followed in actual scientific practice. Popper's rules thus stand refuted by the empirical evidence, and should be rejected.

This line of argument is just not cogent. For it is only to be expected that, at the very least, some scientists will sometimes follow bad methodological policies. And it is possible, despite the apparent enormous success of the empirical sciences, that most scientists have most of the time followed not the best of methodological policies. Thus the fact that a theory of method does not "square" with scientific practice does not in itself constitute a refutation of the theory-although of course it may lead us to suspect that there is something wrong with the theory.

A methodological theory simply does not assert that scientists in fact follow such and such methodological rules of appraisal. Rather a methodological theory should, I suggest, specify (a) a fundamental aim, or group of aims, for science, and (b) a set of methodological rules; it should then assert:

(1) The specified aim is the most worthwhile aim for science that is, as far as we know, in principle realizable.

(2) The specified methodological rules give us the best hope of realizing the specified aim.

In criticizing a theory of method it is these assertions, (1) and (2), that need to be criticized. The fact that the specified methodological rules are not followed in practice does not in itself undermine either (1) or (2).

One slight qualification needs to be added to this. A theory of scientific method, in order to be 
acceptable, must have at least some contact with scientific practice, with the aims and appraisals of working scientists. A theory of method that has no such contact whatsoevereven though it is perfectly satisfactory in all other respects-could be dismissed out of hand as being irrelevant to even the most liberal idea of what constitutes scientific enquiry.

I do not think however that anyone would want to dismiss Popper's theory in this cavalier fashion.

It might be asked: Suppose an internally consistent methodological theory is developed which is closer to scientific practice than Popper's theory is. Should we automatically prefer the rival theory to Popper's theory?

The answer is no. The rival theory may be based on an aim for science which we may consider to be not so worthwhile as the aim for science proposed by Popper. ${ }^{2}$

A failure, then, of Popper's theory of method to reflect accurately scientific practice does not in itself amount to a flaw in that theory: we may, however, take it as a hint that there may be something wrong with the theory. Here we are making the working assumption (which may well be false after all) that scientists in practice mostly adopt the very best methodological policies.

An entirely different, and rather more valid, type of criticism of Popper's theory, that has been developed by Feyerabend [4], Lakatos (see Lakatos' paper in [7]), and at least by implication by Kuhn [5], amounts to the claim that Popper's methodological rules do not give one the best hope of realizing Popper's aims for science. Situations arise, it is argued, in which following Popper's methodological rules would seriously impede the growth of science.

Almost all of Popper's methodological rules have been criticized in this way. Here are in turn some main Popperian methodological rules (see [8]) and, very briefly, the criticisms which have been made of them.

(1) An acceptable new theory must always have greater empirical content than its predecessors.

This is too severe. In certain circumstances it will be in our interests to accept a new theory which initially has far less empirical content than its predecessors, precisely because the new theory promises, with development, with the addition perhaps of auxiliary hypotheses, to lead to a theory of far greater empirical content than its predecessors. ${ }^{3}$ The new theory may for example solve outstanding theoretical problems that the old theories were unable to solve.

It is in any case important to develop rival theories to the existing theories, even if these rivals have nothing like the empirical content of the existing theories, for often it is only by developing such rival theories that we can test the existing theories. ${ }^{4}$

(2) An acceptable new theory must at least be able to explain all of the past success of its predecessors.

Even this more modest demand is too severe. A new theory may be acceptable even though it cannot explain much that its predecessors could explain, for again the new theory may solve severe outstanding problems, and thus promise to lead to a theory which in the end explains all that the old theories explained, and much more besides.

In his later writings Popper has stressed the importance of seeing scientific enquiry as a problem solving activity. Neither Popper nor any of his critics has, however, realized that the demand that a new acceptable theory should solve outstanding problems may conflict with and, on the short-term, actually override the demand that a new acceptable theory should have excess content over its predecessors. 
(3) Always test a theory as severely as possible.

This assumes that in testing a theory our invariable concern is to seek to falsify it. But this assumption is false. In testing a new theory in particular our concern may be to develop the theory, extend the range of its successful applications, build up auxiliary hypotheses. And in order to do this it may well be in our interests to test initially only the most straightforward, least problematical implications of the new theory. We may be justified in actually ignoring, for a time, refuting instances of a theory. For even if we have good grounds for suspecting a theory to be false, it may be in our interests to develop the theory further, as this may indicate more clearly what a new theory must ultimately explain. There is, in short, a methodological point to what Kuhn has called "normal" science. "Revolutions" in science may only be profitable after a phase of normal science [5].

In short, our concern ultimately is to test severely all our theories. But it is theories that have been allowed to develop, grow and reach their full strength that we wish to test severely. It may well be against our interests to test severely, and perhaps eliminate, a promising theory which has not been allowed to come to full fruition.

There is another point. As Lakatos [7] has pointed out, in a research program, refutations may be completely unsurprising and in a way expected, because, to begin with, simplifying assumptions, known to be false, are made. It maybe not the refutations, but rather the corroborations, which are unexpected and surprising. Lakatos considers the following assumptions made by the Newtonian program to explain in detail the motions of the planets.

(i) Masses of heavenly bodies are concentrated at their centres in mass points

of infinite density,

(ii) The sun is stationary,

(iii) The planets do not interact gravitationally.

(iv) There are no tidal effects.

These assumptions were successively dropped as the program became more and more sophisticated. The point to note is that each of these assumptions is actually inconsistent with Newton's laws of motion plus the law of gravitation. If the laws are true, then these simplifying assumptions must be false. In these circumstances there is in a sense no point in testing severely an early crude application of Newtonian theory to the solar system. What is needed rather is the gradual development of the Newtonian program (in this case the development needed was largely mathematical) so that eventually a sophisticated version of Newtonian theory can be severely tested, a version that does not incorporate inconsistent assumptions.

(4) An experimentally "refuted" theory must be rejected.

As before this is too drastic. In general it will not be in our interests to reject a theory that, in the past, has had considerable empirical success until there is an alternative more promising theory on the horizon (see Lakatos' paper in [7]).

(5) An experimentally "refuted" and rejected theory must not be revived at a later stage.

A true theory (or at least an extremely valuable, promising theory) may be "refuted" and quite properly rejected, the "refutation" being due to false auxiliary hypotheses. In order to take this possibility into account, the Popperian rule must be rejected. We can, however, say this: we cannot simply return to an earlier theoretical situation. A theory that has been discarded can only be reaccepted if auxiliary hypotheses, that have been developed subsequently, considerably increase the empirical content of the theory. 
(6) An inconsistent theory cannot be accepted.

It may well be in our interests to accept provisionally a formally inconsistent theory, in the hope that further theoretical work will remove the inconsistency.

It may be asked : Granted that all these criticisms are cogent, do they not effectively demolish Popper's theory? Instead of trying to patch up Popper's theory in the light of these criticisms, should we not reject the theory altogether, and try to find some new and more adequate methodological theory?

As I see it, the situation is like this. Each of the above objections, (1) - (6), is in effect a particular illustration of the general point that if we wish to follow Popper's rules in the long run, then, in certain circumstances, we will be well advised to break these rules on a short term basis. Each criticism argues in effect that it is against our interests to enforce too rigidly Popper's essentially long-term strategic rules on the short-term, tactical level.

But this sort of situation is almost bound to occur given almost any complex, long-term, goal-directed activity. Long-term and short-term interests are almost bound to clash at times. It is to be expected that strategic interests will at times make desirable modes of action which, if judged on a purely short-term, tactical level, would seem to be highly undesirable. Or, in other words, given almost any complex goal-directed activity, it is to be expected that it will be against our interests to enforce too rigidly strategic rules on the tactical level.

It is thus no inadequacy whatsoever in Popper's strategic rules that they too suffer from this quite general complaint. It would in fact be quite extraordinary if they did not.

The above criticisms, (1)-(6), do not effectively demolish Popper's position. They simply spell out in detail the general point that short-term exceptions can invariably be found to long-term methodological rules. It is precisely this general point that Popper has failed to recognize, and it is this failure which vitiates the presentation of his theory.

It is not altogether surprising that Popper has ignored this point. In [8], a major concern of Popper is to solve the problem of demarcation. Consequently, Popper is in the main concerned with those large scale, strategic methodological rules in terms of which (a) scientific enquiry can be distinguished from other types of enquiry; (b) criteria of overall scientific growth can be formulated. Popper, quite understandably, does not stop to consider the detailed application of his methodological rules on the "tactical" level.

Popper's position, then, needs to be modified in the light of the criticisms (l)-(6). ${ }^{5} \mathrm{But}$ this modification does not amount to an ad hoc patching up of Popper's theory. Rather the modification is one which any first formulation of a methodological theory would have to undergo, whatever the game might be - science, war, chess, economic planning, or whatever.

III

I come now to my own major criticism of Popper's theory. It amounts to this: Popper has failed completely to provide any kind of rationale for the methodological rules he advocates. That is, he has failed to provide us with any reason for holding that Popperian rules give us a better hope of realizing the aims of scientific enquiry than any other set of rules. Nor is it easy to see how this failure can be made good within a general Popperian framework. Consequently, Popper has failed to solve his fundamental problem - the problem of demarcation. He has also failed to exhibit science as a rational enterprise. For in order to do this, it is not enough simply to specify an aim for science and a set of methodological rules: we need in addition some 
reason for holding that the rules give us a better hope of realizing the aim than any other set of rules.

In order to develop this criticism, I want to begin by considering-how Popper himself tackles his fundamental problems-which I take to be the problems of demarcation and induction.

The traditional problem of induction, as considered by Popper, can be put like this: How is it possible, by verifying particular instances of a strictly universal statement, or scientific law, to verify the universal statement or law itself, with at least some degree of probability greater than zero ? The problem of demarcation, again as considered by Popper, can be put like this: What is the distinctive feature of scientific theories which enables us to distinguish scientific theories from other sorts of theories ?

With respect to these two problems, Popper's position, put crudely, amounts to this: the distinctive and especially valuable feature of scientific theories is that they are experimentally falsifiable. Falsifiable theories are especially to be prized just because we can discover that they are false; in this field we can detect error, learn from our mistakes, and so hopefully make progress. Scientific theories cannot be verified, or even rendered probable, by any amount of experimental evidence: the traditional problem of induction, in other words, is insoluble. But this does not matter, as long as we give a non-inductivist, non-justificationalist solution to the problem of demarcation. It is only if we hold that the distinctive and especially valuable feature of scientific theories is that they are amenable to inductive verification that it becomes an urgent matter to solve the problem of induction.

In short, Popper offers a solution to the problem of demarcation which, he maintains, makes it unimportant that the traditional problem of induction is insoluble. ${ }^{7}$ Now the point that needs to be emphasized is this. A major part of the tremendous power and suggestiveness of Popper's proposal is due to the fact that Popper's demarcation criterion enables us to explain why we should value scientific theories even though they remain utterly improbable conjectures. The Popperian revolution is, in other words, to a considerable extent, a revolution in values. Before Popper scientific theories were valued for their supposed relative certainty, security, high probability. After Popper, scientific theories are valued for their amazing content and explanatory power, and for their vulnerability to one of the most devastating forms of criticism yet to be discovered - experimental testing. And those scientific theories that we continue to accept are valued for their ability to survive all our most searching attempts to overthrow them.

It is clear then from his solution that Popper interprets the problem of demarcation as the problem of providing a criterion of "scientificness" which enables us to explain why we especially prize scientific theories. A criterion of demarcation which gave no hint whatsoever of why we should value scientific theories more than other sorts of factual theories would be an utterly unacceptable solution to the demarcation problem.

The demarcation problem that Popper sets out to solve-but does not quite succeed in formulating - can, then, be put like this: What is the distinctive feature of scientific theories which (a) enables us to distinguish scientific theories from other sorts of theories, (b) leads us especially to prize scientific theories?

We are now in a position to consider the fundamental problem: Can we provide a rationale for Popper's revised methodological rules ? Do we have any reason for holding that Popper's revised methodological rules give us a better hope of realizing the fundamental aim of science than any other set of rules? 
In order to discuss this question we need to specify precisely what we take to be the fundamental aim of science.

Four "Popperian" aims for scientific enquiry may be distinguished, graded here in order of ambitiousness.

(1) Successively to put forward, and reject, theories in accordance with the methodological rules of acceptance and rejection specified by Popper.

(2) To put forward, and in turn falsify, theories of ever increasing content, which explain all of the empirical success of their predecessors.

(3) To put forward theories which, in addition to (2), successfully predict phenomena not known at the time of their formulation, before they are eventually refuted.

(4) To put forward theories of ever increasing degrees of verisimilitude.

Now if aim (1) is accepted, Popper's methodological rules follow analytically from the conception of science implicit in (1). Anyone who does not adopt Popper's methodological rules is simply not playing the scientific game as defined by (1). In this case, of course, there is no need to provide a rationale for the methodological rules in question. But a high price is paid: this line of approach cannot provide an adequate solution to the problem of demarcation. For, as we have seen, in order to solve the problem of demarcation it is not enough simply to specify necessary and sufficient conditions for a type of enquiry to be scientific: in addition one needs to show why we are justified in especially prizing the theories of a mode of enquiry that is scientific in the required sense. But clearly, the mere fact that a mode of enquiry proceeds in accordance with Popper's acceptance and rejection rules provides no reason whatsoever for especially valuing its theories.

If on the other hand aim (2) is accepted, a provisional reason can be given for especially prizing scientific theories. For one can say of an unrefuted scientific theory that despite the fact that it is vulnerable to refutation, nevertheless it has survived out most earnest attempts to refute it, and thus, prima facie at least, deserves to be taken more seriously than any theory which has not been subjected to this particularly devastating form of scrutiny. But at once there arises the problem of providing a rationale for our methodological rules, for we can ask the question: Do our rules give us the best hope of testing our theories in the severest way possible ? Do our rules give us the best hope of rejecting a theory only when we have isolated some false implication of the theory?

In The Logic of Scientific Discovery Popper seems to adopt the view that methodological rules simply define the game of science, and require no kind of rationale. ${ }^{8}$ But then he must accept (1) as his aim for science, and he cannot claim to have solved the problem of demarcation. Clearly Popper would not wish to accept either of these things. He must therefore face the problem of providing a rationale for methodological rules.

In fact of course Popper does not wish to defend (2) as a worthy aim for science either (see [10], Ch. 10). Taking (3) or (4) as one's aim for science has the advantage of enabling one to give even stronger potential solutions to the problem of demarcation. Thus if one adopts aim (4), one can say that scientific theories constitute our best efforts so far at discovering the truth. But the penalty for this is that the problem of providing a rationale for our adopted methodological rules becomes all the more severe. For we need to establish that our methodological rules give us a better hope of maximizing the verisimilitude of our theories than any other set of rules.

To put it bluntly: because Popper has failed to provide any kind of rationale for the methodological rules he advocates, he has failed to give an adequate solution to the problem of 
demarcation, and to that version of the problem of induction which he would wish to claim he has solved, namely: What criteria ought to govern our selection of theories if our concern is to realize the fundamental aim of scientific enquiry ? In addition he has failed to show that scientific enquiry can be viewed as a rational enterprise. In order to solve these three problems adequately it is essential to show that the advocated methodological rules give one a better hope of realizing the fundamental aim for science than any alternative methodological rules; and it is just this which Popper has failed to do. ${ }^{9}$

In order to drive this criticism of Popperian methodology home, I need to show in greater detail that no rationale for Popper's methodological rules can be provided within Popper's theory, granted that we take in turn (2), (3), and (4) above as the aim for science. Before I do this, however, I would like now to point out that my criticism of Popper is not simply a reiteration of the well-known criticism, outlined by for example Salmon [13], and Lakatos [6], that Popper fails to show that highly corroborated theories are also the most reliable or trustworthy for technological applications.

Let us consider Lakatos' exposition of this criticism. According to Lakatos, in addition to standard Popperian methodological appraisals (which Lakatos calls acceptability! and acceptability^) we need an additional appraisal—acceptability ${ }_{3}$ — which is a measure of the " 'inductive acceptability", 'trustworthiness', 'reliability', 'evidential support', 'credibility', etc." of a theory ([6], p. 391). We need this additional appraisal solely in order to assess the worth of a theory for, practical, technological purposes. Lakatos remarks: "In formulating Popper's methodology there is no need to refer to acceptability 3 " ([6], p. 392).

Now in [6] Lakatos' criticism of Popper amounts to this: the problem of the acceptability of theories is both an important problem, and a problem which Popper fails to solve. With this I entirely agree. (It is just this point that I made in footnote 7 above.) This argument of Lakatos is, however, quite different from the one that I am developing in the present section. For my argument here is this: quite aside from Popper's failure to solve the acceptability 3 problem (which—as Lakatos notes-Popper regards as "comparatively unimportant"), Popper fails, much more seriously, in his primary purpose; namely, to solve the problem of demarcation. A major concern of Popper is to exhibit science as rational enquiry (leaving aside the additional issue of whether science can be shown to be a basis for rational action); and it is just this, I wish to argue, which Popper fails to do; for, he fails to provide a rationale for the methodological rules he advocates. Thus Lakatos criticises Popper for failing to solve a problem which Popper did not really set out to solve in the first place; whereas I wish to criticize Popper's methodology for failing to achieve its primary objective, namely to exhibit scientific enquiry as more rational than other types of enquiry and thus provide a solution to the problem of demarcation.

I want now to discuss in more detail the problem which confronts us in attempting to provide a rationale for Popperian methodological rules, given that our aim for science is in turn (2), (3), and (4) above.

Let us, to begin with, take (2) as our aim for science; that is, let us assume our aim is:

(2) To put forward, and in turn falsify, theories of ever increasing content, which explain all of the empirical success of their predecessors.

Can we show that suitably revised Popperian rules give us the best hope of rejecting a theory only when we have detected some error in that theory?

In view of the Duhemian argument, we cannot hold that an individual theory can be decisively falsified experimentally. It might be, however, that Popperian rules give us the best hope of 
detecting error in our theories.

Consider the following situation. There are two rival research programs, centered round the two conflicting theories $T_{1}$ and $T_{2}$. The first research program, based on $T_{1}$, has for a long time stagnated; a host of auxiliary hypotheses have been proposed to salvage $\mathrm{T}_{1}$ from rejection; despite this, a great number of well-corroborated hypotheses conflict with $\mathrm{T}_{1}$ plus auxiliary hypotheses. In addition this research program has come up with no striking new predictions which have been corroborated.

In contrast to this, the research program based on $T_{2}$ goes buoyantly ahead. The empirical content of $T_{2}$ far exceeds that of $T_{1}$. $T_{2}$ has made a number of striking new predictions which have been corroborated.

In these highly exaggerated circumstances it is clear that Popperian rules would oblige us to accept $\mathrm{T}_{2}$ and reject $\mathrm{T}_{1}$.

Do we have any grounds, however, within Popper's general viewpoint, for maintaining that $T_{1}$ is false ? Do we have any rational grounds for preferring the conjecture that we have detected error in $\mathrm{T}_{1}$ to the conjecture that we have detected error in $\mathrm{T}_{2}$ ?

We might argue: $T_{1}$ is, at least, in difficulties; whereas $T_{2}$ is not. Hence it is more rational to suppose $\mathrm{T}_{1}$ is false.

But suppose $\mathrm{T}_{1}$ is true-something which is perfectly possible. Suppose further that we have adopted some non-Popperian methodology which obliges us to accept $T_{1}$ and reject $T_{2}$, in the above circumstances. In this case it would be $T_{2}$ that would be in very severe difficulties, since it would be "refuted" relative to our acceptance of $\mathrm{T}_{1}$. In these circumstances, adoption of the non-Popperian methodology would lead us to detect genuine error in $\mathrm{T}_{2}$, whereas adoption of the Popperian methodology would lead us to suppress the refutation of $\mathrm{T}_{2}$.

We might argue: the research program based on $\mathrm{T}_{1}$ has stagnated, whereas the research program based on $T_{2}$ forges ahead. It is more rational in these circumstances to suppose that $\mathrm{T}_{1}$ is false, rather than $\mathrm{T}_{2}$.

But why? Perhaps the universe is so constructed that those research programs which forge brilliantly ahead are precisely those which plunge us deeper and deeper into error. It might be some utterly stagnated program which contained the seeds of truth, and which, after further great effort, might enable us to glean a little more of the truth. Popper provides us with no rational grounds for excluding such a possibility.

Finally we might argue: in the above circumstances $T_{2}$ has been more highly corroborated than $T_{1} ; T_{2}$ thus gives us a firmer assurance of being true than $T_{1}$.

But no Popperian can employ this argument, since to do so is to resort to the despised inductive approach to scientific method.

I conclude that, within the general Popperian standpoint, no reason can be given for holding that Popperian rules give us the best hope of rejecting individual theories only when we have detected error in them.

Individual theories cannot be decisively refuted; suitable conglomerations of theories-so it may be argued - can, however, be decisively refuted. It may be held, then, that Popperian rules give us the best hope of detecting error in conglomerations of theories.

But even this more modest claim cannot it seems be maintained. For according to Popper a suitable conglomeration of, let us say, relatively high-level theories is only refuted relative to the acceptance of low-level, experimental, falsifying hypotheses. And these falsifying hypotheses, however well-corroborated, cannot, according to Popper, be established with any more certainty than the high-level theories. Thus we do not have decisive refutations of even 
conglomerations of theories.

We do not even have any reason for supposing that Popperian rules give us the best hope of detecting error in conglomeration of theories. For suppose an enormous number of extremely well-corroborated, low-level, experimental hypotheses conflict with some conglomeration of theories. In this case, if all hypotheses are equally improbable, why not regard the conglomeration of theories as refuting the experimental hypotheses, instead of the other way round? If all that we have is two sets of hypotheses that contradict each other, we can have no reason for preferring the conjecture that error has been detected in one set rather than in the other set.

In reply to this a Popperian might argue that a falsifying hypothesis may be a singular existential statement, which can be established with more security than any universal statement. Thus refutations, if not exactly decisive, are nevertheless reasonably assured by Popperian rules. In order to refute 'All ravens are black' for example we only need to establish: 'There is a family of white ravens in the zoo at New York' (Popper's own example, see [8], p. 87). This falsifying hypothesis is intersubjectively testable; it describes what is in a sense a repeatable effect - since the hypothesis does not simply assert There is a white raven at such and such a time and place'. Nevertheless the hypothesis is a singular statement. ${ }^{10}$

There are a number of objections to this line of argument. Consider the law 'All pieces of copper expand when heated', and suppose a particular piece of copper is discovered which fails to expand when heated. (For simplicity we consider an individual law rather than a conglomeration of laws.)

Now in the first place the statement 'This piece of copper fails to expand when heated' would appear to incorporate universality to as great a degree as any rather more straightforward more universal statement. For the statement asserts, in effect, 'At all times, and in all places, this piece of copper fails to expand when heated'. Hence this statement would appear to be, on Popperian grounds, as impossible to verify as any straightforward universal statement.

To this it may be retorted: but the falsifying hypothesis can quite easily be transformed into a genuinely singular statement: e.g. it could be formulated as 'During the next year (or ten minutes), anywhere on the earth's surface (or anywhere in this laboratory) this piece of copper will fail to expand when heated'.

One might query whether even this severely circumscribed falsifying hypothesis can be verified with any more certainty than any universal statement, since the hypothesis still carries implications about an indefinite number of experiments. But let that pass. ${ }^{11}$ The important point to notice is that, given this last formulation, the hypothesis would not in fact be sufficient to overthrow the physical law 'All bits of copper expand when heated'. For in accepting experimental results as refuting a theory, one is committed to the possibility of explaining these results by some future theory. That is, one is committed to holding, at least as a conjecture, that the refuting experimental results constitute lawful occurrences. For if one denies this conjecture one thereby accepts experimental results which no future physical theory can conceivably explain-since it is only lawful occurrences that can be explained physically. Clearly, physics will be highly resistant to accepting the existence of such phenomena. But to assert that a set of experimental results constitute lawful occurrences is in effect to assert a somewhat vague universal hypothesis. Thus singular hypotheses, however well-corroborated, do not suffice to refute a theory; it is only singular hypotheses, backed up by a universal hypothesis, to the effect that the experimental results in question constitute lawful occurrences, that can refute a theory-or rather a conglomeration of theories. 
Consider again the law 'All bits of copper expand when heated'. Let us suppose This piece of copper, on the surface of the earth, for one year, fails to expand when heated' is highly corroborated within the specified limits of space and time, but that outside these limits the piece of copper in question expands normally when heated. Suppose also that, apart from the above exception, 'All bits of copper expand when heated" continues to be corroborated.

Given these somewhat extraordinary circumstances, we might well decide to retain the law 'All bits of copper expand when heated', and simply declare that in the case of the bit of copper which, for a time, failed to expand when heated, something was going on which we do not understand, and cannot as physicists take into account. That this might be a legitimate decision, in these circumstances, indicates that the singular statement 'This piece of copper, for a time, fails to expand when heated' cannot, however well-corroborated, in itself conclusively refute 'All bits of copper expand when heated'.

However, in the above circumstances we should not I think be obliged to take this line. For we could always conjecture that the anomalous bit of copper was, during the relevant time, in an unusual state, or exposed to unusual conditions, and in the light of this conjecture, reject 'All bits of copper expand when heated". But the crucial point to note here is that we should be rejecting 'All bits of copper expand when heated', not as a consequence simply of accepting the singular hypothesis 'This bit of copper, for a time, fails to expand when heated', but rather as a consequence of accepting the somewhat vague, universal hypothesis 'All bits of copper, when in some specific unknown state, fail to expand when heated'. It is only if we accept tentatively some such hypothesis as this that the behavior of the anomalous bit of copper can be seen as constituting lawful occurrences, which we may hope to explain by means of some future theory.

Of course 'All bits of copper, when in some specific unknown state, fail to expand when heated' is, in Popper's terminology, a metaphysical hypothesis. But this does not affect the argument. Acceptance of this "metaphysical" hypothesis does not mean that the hypothesis is accepted as a full-fledged respectable scientific theory; rather, it means that one is committed to a certain research program, namely to develop a precise, non-metaphysical version of the vague metaphysical hypothesis. If this research program is wholly unsuccessful then we must reject the ostensible refutation of the law 'All bits of copper expand when heated', especially if acceptance of this law leads to a highly successful research program. All of which shows that "This piece of copper, at such and such times and places, fails to expand when heated" however well-corroborated, cannot on its own, conclusively refute 'All bits of copper expand when heated*.

I conclude that no conglomeration of theories can be conclusively refuted, and more generally, that we have no grounds for maintaining within the general Popperian viewpoint, that Popper's methodological rules give us the best hope of detecting error in either individual theories, or in any conglomeration of theories. ${ }^{12}$

As I have already remarked, Popper does not accept that it is sufficient for science to aim at putting forward theories of ever increasing content which are in turn refuted (see [10], Ch. 10). He holds that science must in addition aim at putting forward theories which have a certain measure of empirical success before they are eventually refuted. If we drop the aim of refutation, we may consider the following somewhat revised Popperian aim for science, a revised version of aim (3) above:

(3)' To put forward theories of ever increasing content, which (a) explain the empirical success of their predecessors (b) make successful new predictions before running into severe empirical difficulties. 
Can we maintain that revised Popperian rules, or let us say, Lakatosian rules, give us the best hope of realizing this aim for scientific enquiry? This is not an easy question to discuss, because of a crucial ambiguity in the aim specified in (3)'. Terms such as 'refutation', 'corroboration', 'empirical success', 'successful prediction", and 'empirical difficulties' can be interpreted in two radically different ways. On the one hand these terms may be explicated solely in terms of certain adopted methodological rules, no attempt being made to provide a rationale for these rules. In this case 'refutation', for example, simply means that the methodological rules require that we reject the theory in question. Again, 'successful prediction' means simply that the theory in question predicts a hypothesis which our rules require us to accept. We may call this the "methodological" interpretation of the above terms.

On the other hand the above terms may be given what we might call an "epistemological" interpretation. Here the assumption is that we have specified some aim for science which enables us to explain why we especially prize scientific theories, and hence which enables us to give an adequate solution to the problem of demarcation. 'Refutation', 'successful prediction', etc., are then explicated in terms of this aim. Thus the epistemological interpretation of 'refutation' might be that we have good, or rational, grounds for holding the theory in question to be false. The epistemological interpretation of 'successful prediction' might be that the prediction should be taken seriously in that it has survived our very real attempts to falsify it.

Now if the relevant terms in (3)' above are given a methodological interpretation only, then it is clear that the aim specified in (3)' cannot constitute a satisfactory aim for science. For such an aim amounts to no more than the aim to accept and reject theories in accordance with Popperian, or Lakatosian, rules of acceptance and rejection. In terms of such an aim we can give no explanation of why we especially prize scientific theories; we cannot provide an adequate solution to the problem of demarcation.

If on the other hand we interpret the relevant terms of (3)' epistemologically, then all the old problems arise afresh. Either we give a non-Popperian, justificational interpretation to the relevant terms, or we give a Popperian interpretation, which commits us to maintaining that Lakatosian rules give us the best hope of detecting error in our theories. And this, we have already argued, cannot be maintained. There just does not seem to be a third interpretation available. If 'refutation' has nothing to do with the desired detection of falsehood, and 'corroboration' has nothing to do with the desired detection of truth, then it seems we have abandoned what I have called the "epistemological" realm altogether. Scientific investigation becomes simply an intricate game, the only purpose of which is to play the game in accordance with the rules. ${ }^{13}$

One might of course attempt to give an extra-methodological point to aim (3)' in terms of the claim that highly corroborated theories are more trustworthy, for technological purposes, than uncorroborated or refuted theories. But apart from the intrinsic difficulties associated with this claim-it involves something like the traditional problem of induction - to adopt this line would be to give essentially an instrumentalist defence of the value of science, which would be to break radically with the Popperian tradition.

There is finally Popper's fourth aim for science to consider, namely:

(4) To put forward theories of ever increasing degrees of verisimilitude. But this case clearly requires no additional discussion. If one cannot even provide a rationale for Popperian rules given the relatively unambitious aims for science, (2) and (3), then quite clearly there can be no hope of providing a rationale for Popperian rules, within Popper's 
general viewpoint, given the highly ambitious aim for science to approach closer and closer to the truth. ${ }^{14}$

There is, however, one reply that Popper might wish to make to my whole argument so far. Popper might argue that it must always be a conjecture that such and such rules give one the best hope of realizing the aim of science-whether that aim is the detection and elimination of error, or the discovery of the truth. One cannot know for certain the methodological rules one adopts are the best available. In this domain too one can only put forward conjectures, and then seek to refute them, by subjecting them to severe criticism.

This reply does not however dispose of my argument. On the contrary, my whole argument can be interpreted as being designed precisely to show that, within Popper's general standpoint, there can be no reason for preferring one conjecture to another as to the best rules to adopt if one wishes to detect error, or progress towards the truth. In other words I have argued, in effect, that within Popper's general standpoint, conjectures about which rules it is best to adopt are all equally uncriticizable. It is in this sense that we have no grounds, no reasons, for preferring one conjecture to another.

\section{IV}

No rationale can then, it seems, be provided for Popperian methodological rules, given Popper's general methodological viewpoint. It is natural to ask: Can a rationale be provided for Popperian rules which does not do violence to the whole spirit of Popper's philosophy, even though it may conflict with one or other of Popper's rather more detailed methodological theses? I am inclined to think that such a rationale can be provided. But first the terms of the discussion need to be changed a little.

So far the problem has been discussed in the following terms. Granted that the aim of science is to progress towards the truth (or, more modestly, to detect and eliminate error) how can we show that methodological rules roughly similar to those advocated by Popper give us a rational hope of realizing this aim ? In my view the problem posed in this way makes the fundamental mistake of supposing that the aim of science is simply to progress towards the truth (at its most ambitious). For the fact is surely that a basic aim of science is to search for explanations. Science seeks, not truth per se, but rather what might be characterized as explanatory truth. That is, science seeks to put forward theories of both increasing explanatory power and increasing verisimilitude. ${ }^{15}$ The possibility that the truth is nonexplanatory is something which science simply disregards, even though we have no good reason to suppose that the truth is explanatory rather than nonexplanatory.

The notion of "explanatory power" or the closely related methodological notion of "simplicity" is of course notoriously difficult to characterize precisely. Roughly one can say that the more a theory predicts, and the fewer the number of independent postulates it has, so the greater is the theory's degree of simplicity, or of explanatory power. A theory which is so "unsimple" as to have as many independent postulates as the number of laws it entails is not explanatory at all.

Now Popper is, of course, ready to acknowledge that a basic aim of science is to search for explanations (see the example [9], and [10], pp. 114-115). However, Popper has repeatedly tried to reduce this aim to an even more fundamental aim. A basic tenet of [8] is that simplicity, or explanatory power, is to be equated with empirical content. And Popper has tried to reduce the aim of searching for theories of ever higher empirical content to the aim either of detecting and eliminating error, or of approaching the truth. In [8] Popper may be interpreted as 
taking the detection and elimination of error as the fundamental aim of science. In order to realize this aim, we need to consider theories vulnerable to refutation, and in particular theories of ever increasing degrees of falsifiability (and of course degree of falsifiability $=$ degree of empirical content). Later Popper argues that if we pursue the fundamental aim of approaching the truth we need to consider theories of ever increasing degrees of empirical content [11]. Thus Popper tries to reduce the search for explanations to a search for high empirical content which is in turn reduced to a search either for elimination of error or for progression towards the truth.

Both these attempts to reduce the aim of seeking explanations to more fundamental aims fail for the simple reason that high empirical content cannot be equated with high explanatory power. ${ }^{16}$ For we can always increase the empirical content of a theory by tacking on independently testable postulates, and this could quite clearly drastically decrease the simplicity, the explanatory power, of the theory in that the number of logically independent postulates would go up.

My suggestion, then, is that we should take the search for explanations-for explanatory systematization - as a fundamental aim of science, and not as a derivative aim; not as an aim to be reduced to some more fundamental aim by means of some such claim as that explanatory theories are more verifiable, more falsifiable, or better candidates for high verisimilitude than nonexplanatory theories. Explanatoriness should be conceived of as an end in itself, and not as a means to some other end.

The situation before us, then, is this. The problem that Popper fails to solve is:

(a) Given that the aim of science is to progress towards the truth (or, more modestly, to eliminate error), how can we show that the methods of science give us the best, the most rational hope of realizing this aim ?

This problem needs, I suggest, to be replaced by the following problem:

(b) Given that the aim of science is to develop theories of both increasing explanatory power and increasing verisimilitude, how can we show that the methods of science give us the best, the most rational, hope of realizing this aim?

Problem (a) is insoluble for the following fundamental reason. Physics places a premium on theories of extreme simplicity, of high explanatory power. No reason can be given, however, for supposing that simple theories as opposed to complex theories are either nearer to the truth or more falsifiable. If the world is in fact extremely complex so that in the end no explanation can be given for phenomena, then the simpler our theories become, the further from the truth they will be. Again, a complex theory of high empirical content is strictly just as falsifiable as a simple theory of equal empirical content. Thus, given either the aim of developing theories of increasing falsifiability (i.e. the aim of increasingly efficient error elimination) or the aim of approaching the truth, no rationale can be provided for those methodological rules which place a premium on simple theories, on theories of high explanatory power.

This fatal difficulty does not, however, arise in connection with problem (b). Given that a fundamental aim of science is to search for explanations no difficulty arises in providing a rationale for those methodological rules which favor explanatory theories to nonexplanatory theories.

This easy victory is of course won at a price. Problem (b) is solved at the expense of creating a new problem. For, of course, in order to exhibit science as rational enquiry (and thus 
adequately solve the demarcation problem) we need to show not only that the rules of science are rational, but also that the aim of science is a rational aim to adopt. We thus have the new problem:

(c) How can we show that the aim of seeking theories of both increasing explanatory power (or simplicity) and increasing verisimilitude is a rational aim to adopt?

Before considering problem (c), however, I wish to discuss in a little more detail my solution to problem (b). We can, I suggest, argue as follows. As long as we are pursuing the aim of seeking explanatory truth, it will be entirely rational to plan our strategy on the assumption that our search will meet with success. But our search can only meet with success if the world does ultimately have a simple structure, or, in other words, if a certain metaphysical thesis-let us call it the thesis of "structural simplicity" - is true. For if the world is ultimately incredibly complex, then as our theories increase in explanatory power, or simplicity, they will move further and further away from the truth. Therefore, as long as we seek explanatory truth, it will be entirely rational to base our strategy on the assumption that the thesis of structural simplicity is true.

It may be asked: But how can it possibly be rational to make such an incredibly risky assumption as the thesis of structural simplicity when we have no reason whatsoever to suppose this thesis to be true? That we have no reason to suppose the thesis to be true I grant. The crucial point however is this: As long as our aim is to seek explanatory truth it is only risky to make those assumptions which, if wrong, threaten to endanger the success of our enterprise. But in assuming the thesis of structural simplicity to be true we risk nothing since the truth of this thesis is just a condition for our enterprise to be successful. Making the assumption that the thesis of structural simplicity is true can in no way whatsoever endanger or block the success of our enterprise. If this assumption is false, then there is no way whatsoever in which we can realize our goal, whether we make the assumption or not. Thus making the assumption adds no additional risk to the success of our enterprise whatsoever. ${ }^{17}$ (It is only relative to the search for truth per se that the assumption becomes incredibly risky.)

Granted, then, that we are pursuing the aim of seeking explanatory truth we may entirely rationally, and wholly without risk, plan our strategy on the assumption that the thesis of structural simplicity is true, even though we have no reason whatsoever to suppose that this thesis is true. But if the thesis of structural simplicity is true, then it is only reasonable to suppose that the regularities we observe in the world - the low-level hypotheses that we have corroborated-are due ultimately to the structural simplicity of the universe. As physicists, in seeking more and more accurate delineations of the structural properties of the universe, our best policy must be to develop high-level, bold conjectures which explain as much of the observed regularities - the low-level corroborated hypotheses-as possible. When our highlevel theory conflicts with a number of well-corroborated low-level hypotheses, and when our attempts at patching up our theory lead to considerable theoretical complexities, it is only rational to suppose that our original high-level theory is on the wrong lines. We must look for a new theory, involving fewer independent postulates, which can explain all that the old theory failed to explain. In particular, it will be rational to choose a new theory which successfully predicts a number of striking new phenomena: for such a theory holds the promise of delineating quite accurately some aspect of the ultimate structure of the universe.

This argument, if successful, only establishes the rationality of adopting roughly Popperian rules granted that we are pursuing the aim of seeking explanatory truth. But is this a rational 
aim to pursue in the first place? How, in other words, are we to solve problem (c)?

It may be thought that problem (c) is just as insoluble as problem (a). For if the aim specified in (c) is to be rational, do we not need to have some rational assurance that this aim will meet with success? But this aim of seeking explanatory truth can only meet with success if the thesis of structural simplicity is true. And no reason, no rationale, can be given for holding that the thesis of structural simplicity is true rather than false. It looks, then, as if the aim of seeking explanatory truth cannot be a rational aim.

One standard way of attempting to solve the problem of induction, or the problem of providing a rationale for methodological rules, is to introduce a metaphysical thesis which, if true, would explain the success of our inductive inferences, the validity of our methodological rules (see [6] for a discussion of this kind of approach). The obvious objection to such an approach is that it only succeeds if we can provide some reason for holding that the relevant metaphysical thesis is true. If no reason whatsoever can be provided for this, then the introduction of the metaphysical thesis does not help at all. And of course no reason can be given for maintaining that it is more rational to hold that such a metaphysical principle is true rather than false. Thus the familiar attempt to resolve the problem along these lines fails.

But does not the approach that I am advocating here fail for similar reasons? Does not the rationality of the aim of seeking explanatory truth require that it is more rational to hold that the metaphysical thesis of structural simplicity is true rather than false ?

The answer is: no. An aim can be rational even though we have no rational assurance whatsoever that the aim will meet with success. Of course, if we have rational reasons for believing that an aim cannot meet with success, then it is irrational to pursue such an aim. But in the absence of such reasons, it may well be rational to pursue some goal even though we have no reasons in advance for maintaining we will meet with success. This view is enshrined in the adage that one cannot know what one can do until one tries. Perhaps the majority of new projects, initiated by mankind, have been initiated without advance rational assurance of success. Of course if a project meets with no success whatsoever after long effort, then gradually this in itself may be taken as a reason for holding that the project cannot succeed, and is irrational. (Perhaps alchemy might be viewed in this light.)

Thus for the aim of seeking explanatory truth to be rational it is not necessary that it is more rational to hold that the thesis of structural simplicity is true rather than false. All that is required is that it is not positively irrational to hold that the thesis of structural simplicity might possibly be true. It is only if it were more rational to hold that the thesis of structural simplicity is false rather than true that the aim of seeking explanatory truth would fail to be rational.

Thus the approach advocated here successfully overcomes the standard lethal objection to the familiar attempt to provide a rationale for methodological rules by introducing an appropriate metaphysical principle. Problem (c) is solvable even though problem (a) is not.

One final objection, to my claim that the aim of seeking explanatory truth is rational, needs to be considered. It might be objected that I have, in a sense, established too much in that I have established the rationality of any crazy line of enquiry whatsoever.

Consider for example the marvelous fairy tale of scientology. At one time we were all gods. But we grew bored. So we created the universe, and immersed ourselves in it, to keep boredom at bay. But after several reincarnations, we gradually forgot who we were and the powers that we possessed, until we ended up in our present pitiful state, as mere human beings. Therapy can, however, enable us to recover our memory and our powers. 
Now a scientologist can, it seems, argue that the aim of developing successful therapeutic methods is just as rational as the physicist's aim of seeking explanatory truth. What entitles us to claim that the aims of physics are more rational than the aims of scientology?

Ultimately I think we can only say that it is only the enormous apparent success of physics which entitles us to make this judgment. (I say apparent success, for of course we do not know that the aim of seeking explanatory truth, via physical enquiry, has met with real success.) The moment scientology began to meet with a comparable degree of apparent success the scales would begin to tip the other way. If a scientologist, after therapy, was able, merely by the exercise of thought, to shift the planets in their course round the sun, or alter a law of nature or two, then physics might begin to look rather silly as an alternative rational search for explanatory truth.

\section{References}

[1] Deutscher, M. "Popper's Problem of an Empirical Base." Australasian Journal of Philosophy 46 (1968): 277-288.

[2] Duhem, P. The Aim and Structure of Physical Theory. Princeton: Princeton University Press, 1954.

[3] Feyerabend, P. "Problems of Empiricism." Beyond the Edge of Certainty. University of Pittsburgh series in the Philosophy of Science, edited by R. Colodny, vol. 2. Englewood Cliffs: Prentice-Hall, 1965.

[4] Feyerabend, P. "Problems of Empiricism II." The Nature and Function of Scientific Theories. University of Pittsburgh series in the Philosophy of Science, edited by R. Colodny vol. 4. Pittsburgh: University of Pittsburgh Press, 1970.

[5] Kuhn, T. The Structure of Scientific Revolutions. Chicago: University of Chicago Press, 1962.

[6J Lakatos, I. "The Changing Problem of Inductive Logic." The Problem of Inductive Logic. Amsterdam: North Holland, 1968.

[7] Lakatos, I., and Musgrave, A., eds. Criticism and the Growth of Knowledge. Cambridge: Cambridge University Press, 1970.

[8] Popper, K. The Logic of Scientific Discovery. New York: Basic Books, 1961.

[9] Popper, K. "The Aim of Science." Ratio $\backslash$ (1957): 24-35.

[10] Popper, K. Conjectures and Refutations. New York: Basic Books, 1962.

[11] Popper, K. "A Theorem on Truth Content." Mind, Matter, and Method. Edited by P.

Feyerabend and G. Maxwell. Minneapolis: University of Minnesota Press, 1966.

[12] Rudner, R. "An Introduction to Simplicity." Philosophy of Science 28 (1961): 109-

119.

[13] Salmon, W. "The Justification of Inductive Rules of Inference." The Problem of Inductive Logic. Edited by I. Lakatos. Amsterdam: North Holland, 1968.

\section{Notes}

${ }^{1}$ At least a part of Kuhn's case against Popper is of the type: see for example [7], pp. 4-7. Pp. 237-238 also show Kuhn's commitment to the validity alleged of this type of argument. Elsewhere in this book the same type of argument is employed; e.g. by L. Pearce Williams, p. 50, and by Lakatos, p. 115.

${ }^{2}$ Of course a methodologist may be concerned to characterize not, as Popper is, the highest ideal of science, but rather science as it in fact exists. In this case the methodologist will select those fundamental aims for science which are such that the rules best adapted to realize these aims are as close as possible to rules in fact adopted by the majority of scientists. In this case of course the 
"correct" methodological theory will be that internally consistent theory which is closest to scientific practice.

This, on a charitable interpretation, is perhaps Kuhn's conception of methodology.

${ }^{3}$ See [4] for a powerful development of this criticism. Feyerabend, however-in my view quite wrongly - regards this criticism as spelling the downfall of Popper's theory of scientific method.

${ }^{4}$ This point is made by Feyerabend in [3]. One might add that Popper's notions of severe testing, and of corroboration, actually require that when we test a theory we have some rival theory up our sleeve.

${ }^{5}$ Such a modified version of Popper's theory has been put forward by Lakatos in [7].

${ }^{6}$ Popper here conflates three distinct demarcation problems, namely the problems of distinguishing:

(i) Empirical theories from other sorts of theories (metaphysical, a priori, etc.).

(ii) Scientific modes of enquiry from unscientific or pseudoscientific modes of enquiry,

(iii) The highest form of scientific enquiry from other less worthwhile forms of scientific enquiry grading into the pseudoscientific.

A few remarks about these three problems.

Popper clearly is primarily concerned to solve the third of these three demarcation problems. Kuhn, on the other hand, I have suggested, may be interpreted as being concerned to solve the second demarcation problem. And the logical positivists sought, and failed to discover, a solution to the first demarcation problem, in so far as their principle of verification was intended to be a criterion of empiricalness, and not, absurdly, a criterion of meaning.

It seems to me to be desirable to regard 'scientific' as being attributable, in the first instance, to modes of enquiry, rather than, as Popper would have it, to theories, or even as Lakatos would have it, to series of theories. What is most characteristic of science is its methods of investigation, rather than the type of theory it deals with. We can of course then go on to apply 'scientific' to a theory which is proposed as part of the course of scientific investigation.

It also seems to me to be desirable to distinguish an empirical theory from a scientific theory. A theory may be empirical in Popper's sense of being experimentally falsifiable, and yet may not be proposed in the context of a scientific enquiry. We need to be able to say that a mode of enquiry considers only empirical theories, and yet is not scientific: Popper's terminology does not permit this.

It should be noted, however, that a theory can only be said to be empirical or experimentally falsifiable relative to the acceptance of at least two distinct sorts of methodological rules. First, there must be agreement about what sort of statement can constitute a basic statement. Second, there must be agreement about under what circumstances a falsifying hypothesis is to be accepted. These are not necessarily trivial matters. In psychology, for example, there is no general agreement about what sort of statement qualifies as a basic statement.

It may be asked: If only conglomerations of theories are experimentally falsifiable, and not individual theories, how can we define the empirical character of an individual theory in terms of falsifiability ? This can, I think, be done (using the concept of empirical content) along the following lines. A hypothesis $h$ is empirical if, and only if:

(a) There is a falsifiable conglomeration of hypotheses, T, which entails A; and which is such that:

(b) There is no $\mathrm{T}^{\prime}$, with the same empirical content as $\mathrm{T}$, such that $\mathrm{T}$ entails $\mathrm{T}^{\prime}$, but $\mathrm{T}^{\prime}$ does not entail h.

(In other words we cannot omit $\mathrm{h}$ from $\mathrm{T}$ without decreasing the empirical content of $\mathrm{T}$.)

This seems to correspond to our intuitions. For example we are inclined to call Newton's postulate concerning absolute space metaphysical, i.e. non-empirical, precisely because we can remove this postulate from Newtonian theory without thereby decreasing the empirical content of that theory. 
Here then I suggest is a solution to that age-old problem of giving a precise formulation to the so-called principle of verification. Note: I do not wish to suggest that metaphysical components of a scientific theory are necessarily undesirable.

${ }^{7}$ Of course, a rather more general version of the problem of induction may be formulated, which is less obviously insoluble, such as: What criteria ought to govern our choice of a theory from two or more rival theories? (Here we simply drop the implicit assumption that it is high probability which ought to govern our choice of theories.) It at once becomes clear that there are at least two distinct versions of this formulation of the problem of induction, namely:

(i) What criteria ought to govern our choice of a theory from two or more theories if our concern is with scientific growth?

(ii) What criteria ought to govern our choice of a theory from two or more rival theories, if our concern is with the trustworthiness of the theory, for purposes of technological application?

Now Popper does claim, in effect, to have solved the first version of this problem. But about the second version of the problem Popper is altogether silent. He cannot claim to have (a) solved the problem; (b) shown the problem is insoluble; or (c) shown that the problem is unimportant. It might of course turn out that a satisfactory solution of (i) also provides a satisfactory solution of (ii): but this is not obvious. It needs to be argued. (Lakatos in effect sets out to provide something like such an argument in [6].) Popper, however, provides scarcely even a hint of such an argument-apart from the odd stray remark (e.g. [10], p. 51). Here then is an inadequacy in Popper's views in addition to the one discussed in the text.

${ }^{8}$ Popper says for example: "Just as chess might be defined by the rules proper to it, so empirical science may be defined by means of its methodological rules," see [8], p. 54.

${ }^{9}$ It is a certain ambiguity in Popper's notion of "falsifiability" or "refutability" which is in a sense responsible for this failure. Popper holds in effect that scientific theories are not decisively falsifiable - in order to meet the Duhem argument, or "conventionalist objections"-and at the same time holds that scientific theories are decisively falsifiable-in order to hold at bay the rationale problem, and thus ensure that he can give an adequate solution to the problem of demarcation.

${ }^{10}$ One reply to this Popperian counterproposal is to argue, as Deutscher in effect does (see [1 ]), that Popper provides us with no reason for supposing that the methodological rules that govern acceptance of singular existential statements are the best available if we wish only to accept true basic statements. Deutscher is, I think, right in stressing that Popper fails to solve this problem adequately. However, it seems reasonable to suppose that a solution can be found to this problem within Popper's general methodological position. But a solution to this problem would not really help Popper at all. For, as I argue in the text, acceptance of singular existential statements cannot lead to the overthrow of physical theories. It thus, within the Popperian framework, remains utterly problematic how we could ever have good grounds for supposing a theory to be falsified. I do not think, therefore, that Deutscher has isolated the most severe part of the problem that confronts Popper's theory.

${ }^{11}$ Popper could not in fact let this pass, for he holds that even a singular existential statement will involve some law, in that the statement will attribute a dispositional property to some object (see [8], pp. 423-424). I am not so sure, however, that in attributing a dispositional property to an object we are necessarily, if implicitly, asserting some law-statement. It seems to me to be conceivable that genuine singular existential statements, not involving laws, but nevertheless specifying a repeatable, intersubjectively testable effect, are possible.

${ }^{12}$ In order to avoid a possible misunderstanding, I should perhaps add that I believe that something like Popperian rules do give us the best hope of detecting error in our theories. The problem however is to show that we have good reasons for this thesis-especially within Popper's general methodology.

${ }^{13}$ A somewhat similar argument has been developed independently by Lakatos; see Lakatos' 
contribution to the forthcoming Schilpp volume on Popper.

${ }^{14}$ Of possible relevance to the problem of providing a rationale for Popperian rules given the aim of increasing the truth-content of our theories, is Popper's thesis that the only way to increase truth-content is to increase content (see [7], p. 57 and [11]). But this at most provides a rationale for just the rule: put forward theories of ever increasing empirical content. It does not in any way provide a rationale for all the other Popperian rules.

${ }^{15}$ Essentially the same point has been made by Rudner, who writes for example ". . . systematization seems to me as much a desideratum of science as is truth ..." ([12], p. 118).

${ }_{16}$ High empirical content is at best a necessary condition for high explanatory power or simplicity; it is certainly not a sufficient condition. Actually Popper has in effect acknowledged the need for a criterion of simplicity, or explanatory power, which goes beyond the notion of empirical content. For he asserts that we should require of a new theory that it should "proceed from some simple, new, and powerful unifying idea" ([10], p. 241), and goes on to call this a requirement of simplicity, which cannot, it seems, be formulated very clearly. Popper does not, however, go on to argue (as I do in the text) that the search for explanations or simplicity should be taken as a fundamental aim of science, and that this enables us to provide a rationale for both the rules and the aims of science.

${ }^{17}$ One potential reason only exists for considering the possibility that the thesis of structural simplicity is false, granted that we are seeking explanatory truth. If there were the slightest hope of establishing that the thesis of structural simplicity is false, then it would be entirely rational to consider this possibility (so that we can call our whole enterprise off the moment we discover our goal is unobtainable). But there is no hope of establishing that the thesis of structural simplicity is false, any more than there is a hope of establishing that it is true. The best that we can do, by way of showing that the search for explanatory truth cannot succeed, is to pursue this goal as strenuously as possible, and after untold years of effort, fail in the attempt. Thus, granted that we are pursuing the goal of seeking explanatory truth, the only potential reason for considering the possibility that the thesis of structural simplicity is false collapses. 